will provide an escape hatch if the climate ever does reach a tipping point. This does not mean that the discussions should be avoided, but rather that the speculations need to be backed up with a solid body of research. Moreover, geoengineering research should be framed not as a hope for deus ex machina fixes to sudden global deterioration, but as a palliative cushion for the worst excesses of the peak years that are inevitable even after emissions start to be cut. A world slightly shaded from the Sun while its carbon levels are brought down by means of active capture would be a strangely unnatural place - but not necessarily a bad one, compared with the alternatives.

Research on local and regional interventions to cool Earth should be undertaken now - ideally in a way that provides basic information to climate researchers. Most sciences are in the habit of poking that which they study to gauge its response. Climate researchers lack such a tradition, and might have something to gain by starting one up. The attention to far-off goals, however, must not obscure short-term opportunities. In addition to cutting $\mathrm{CO}_{2}$ emissions, global leaders should curb the release of other substances warming the climate, notably methane and soot, also known as 'black carbon'. Tackling such pollutants will bring other benefits, too, such as reducing the respiratory problems associated with cooking over smoky fires and with high levels of tropospheric ozone.

With so many challenges still to be faced, the climate problem may seem insurmountable. But there is still time left to act, and there is hope to be found in human ingenuity. Humans have a long history of finding new ways to tackle problems, and new ways to circumvent the worst. Without commitment from the highest levels, such ingenuity is likely to come to naught. But with such a commitment, and with a worldwide determination to make a serious cut in emissions, there is much that can usefully, and invigoratingly, be done.

\section{Authorship policies}

\section{We are clarifying the duties of lead authors and making author-contribution statements mandatory.}

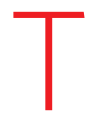
he Nature journals encourage authors to treat their data and their collaborators with the respect that their communities demand. High-profile journals have a duty to reinforce the trends towards greater transparency and to help scientists to fulfil their responsibilities as researchers and authors. We are therefore introducing small but important changes in our policies to reflect these goals.

In a previous Editorial (Nature 450, 1; 2007), we asked for feedback about whether we should require senior or corresponding authors to sign a statement that they had taken some specific 'integrity insurance' steps before the manuscript was submitted. Some applauded this idea, but most were not in favour. (Some of the feedback can be seen at http://tinyurl.com/ddjsxa). Major doubts were expressed about the ability of corresponding authors to take on such responsibility given the diversity of collaborations. The belief was also expressed that such signed statements would too often be worthless box-ticking exercises. Although we regretfully accept these realities, we believe that we should go further in spelling out the responsibilities of co-authors, and in requiring an implicit acceptance of them.

Accordingly, we have modified the Nature journal policy on authorship, which is detailed on our website (http://tinyurl.com/ dkgbf8). For papers submitted by collaborations, we now delineate the responsibilities of the senior members of each collaboration group on the paper. Before submitting the paper, at least one senior member from each collaborating group must take responsibility for their group's contribution. Three major responsibilities are covered: preservation of the original data on which the paper is based, verification that the figures and conclusions accurately reflect the data collected and that manipulations to images are in accordance with Nature journal guidelines (http://tinyurl.com/ cmmrp7), and minimization of obstacles to sharing materials, data and algorithms through appropriate planning.

Corresponding authors have multiple responsibilities, but we now make it clearer that the author list should include all appropriate researchers and no others, and that the order has been agreed to by all authors. They are expected to have notified all authors when the manuscript was submitted, they are the point of contact with the editor and they must communicate any matters that arise after publication to their co-authors.

Another change is that we have strengthened our policy for statements of authors' contributions. This policy was first introduced nearly 10 years ago (Nature 399, $393 ; 1999)$ to make the credit due to individual co-authors more explicit. Since then, authors of Nature papers have had the opportunity to include in their papers a statement that details each author's role in the published work. Over the past 10 years,
"We believe that we should go further in spelling out the responsibilities of co-authors, and in requiring an implicit acceptance of them." the proportion of authors who choose to include this has risen dramatically.

This acceptance, and discussions with authors who have chosen not to include such a statement, has led us to change our policy. Rather than 'strongly encouraging' such statements, we now require them for publication of original research papers in Nature and the Nature research journals. The detail provided can vary tremendously (http://tinyurl.com/39mmyw) and authors are left to structure them as they see fit. We insist only that no author be left out.

To ensure that authors are familiar with these changes, we will shortly require the corresponding author to confirm that he or she has read the Nature journal policies on author responsibilities (http://tinyurl.com/dkgbf8) and is submitting the manuscript in accordance with those policies. 